\title{
Semiconducting Electronic Property of Graphene Adsorbed on (0001) Surfaces of $\mathrm{SiO}_{2}$
}

\author{
Nguyen Thanh Cuong, ${ }^{1,3}$ Minoru Otani, ${ }^{1,3}$ and Susumu Okada ${ }^{2,3}$ \\ ${ }^{1}$ Nanosystem Research Institute (NRI), National Institute of Advanced Industrial Science and Technology (AIST), \\ Tsukuba 305-8568, Japan \\ ${ }^{2}$ Graduate school of Pure and Applied Sciences, University of Tsukuba, \\ 1-1-1 Tennodai, Tsukuba 305-8571, Japan \\ ${ }^{3}$ Japan Science and Technology Agency, CREST, 5 Sanbancho, Chiyoda-ku, Tokyo 102-0075, Japan \\ (Received 8 September 2010; revised manuscript received 7 January 2011; published 7 March 2011)
}

\begin{abstract}
First-principles total energy calculations are performed to investigate the energetics and electronic structures of graphene adsorbed on both an oxygen-terminated $\mathrm{SiO}_{2}$ (0001) surface and a fully hydroxylated $\mathrm{SiO}_{2}$ (0001) surface. We find that there are several stable adsorption sites for graphene on both O-terminated and hydroxylated $\mathrm{SiO}_{2}$ surfaces. The binding energy in the most stable geometry is found to be $15 \mathrm{meV}$ per $\mathrm{C}$ atom, indicating a weak interaction between graphene and $\mathrm{SiO}_{2}(0001)$ surfaces. We also find that the graphene adsorbed on $\mathrm{SiO}_{2}$ is a semiconductor irrespective of the adsorption arrangement due to the variation of on-site energy induced by the $\mathrm{SiO}_{2}$ substrate.
\end{abstract}

DOI: 10.1103/PhysRevLett.106.106801

Exfoliation of graphite [1,2] and thermal annealing of $\mathrm{SiC}[3,4]$ have now achieved the single-layered graphite, graphene, which provides us a perfect two-dimensional electronic system. Much effort has been devoted not only to the elucidation of fundamental physics on this new and famous carbon allotrope but also to the exploration of the possibility of application in the next generation of electronic devices [1,5-9]. Graphene possesses a pair of linear dispersion bands at the Fermi level resulting in remarkable carrier mobility of a few hundred thousand $\mathrm{cm}^{2} / \mathrm{V} \mathrm{s}$, and leading to application of graphene in high-speed switching devices [1,5-10]. On the other hand, these remarkable electronic properties of graphene have been shown to be vulnerable to external conditions such as structural corrugations, atom or molecule adsorptions, and interactions with other graphene or substrate surfaces [8-13]. Although this issue may be fatal to the application of graphene as a switching device in semiconductors, graphene is still applicable as a constituent of sensing devices. From both the fundamental precise two-dimensional physics and applied semiconductor engineering points of view, it is important to elucidate the effects of other materials on the electronic and structural properties of graphene.

Hybrid structures of graphene with insulating substrates are important for both fundamental studies and applications of graphene. Silicon dioxide $\left(\mathrm{SiO}_{2}\right)$ is widely used as an insulating substrate for device fabrication and physical property measurement. Many experiments reported the modulation of the electronic properties of graphene on a $\mathrm{SiO}_{2}$ substrate, such as band gap opening and decrease of carrier mobility [8-10]. The interaction between the graphene and substrate is considered to play a crucial role in the modulation. Although many theoretical calculations [14-16] have been performed to elucidate the fundamental properties of graphene adsorbed on $\mathrm{SiO}_{2}$ surfaces, there is
PACS numbers: 73.22.Pr, 61.48.Gh, 71.15.Mb, 81.05.ue

still no clear physical insight into the fundamental properties of this hybrid system. Therefore, in the present work, we aim to investigate theoretically the energetics and electronic structures of graphene adsorbed on (0001) surfaces of $\alpha$ quartz using realistic and physical surface atomic structures based on first-principles total energy calculations in the framework of density functional theory. Our calculations clearly show that graphene keeps its plane structure, and is weakly bound to the substrate without forming covalent bond with $\mathrm{O} / \mathrm{Si}$ atoms. The calculated binding energy calculated is about $10 \mathrm{meV}$ per $\mathrm{C}$ atom. We also found that the graphene has semiconducting properties with a direct band gap of a few tens of meV. Detailed analysis of the local electrostatic potential reveals that the semiconducting properties are ascribed to the modulation of on-site energy of $\mathrm{C}$ atoms induced by the substrate.

All calculations have been performed based on density functional theory $[17,18]$. We use the local density approximation (LDA) to express the exchange-correlation energy of interacting electrons $[19,20]$. Ultrasoft pseudopotentials are adopted to describe the electron-ion interaction [21]. The valence wave functions and charge density are expanded in terms of a plane-wave basis set with cutoff energies of 25 and $225 \mathrm{Ry}$, respectively. The Brillouinzone integration is performed with a $6 \times 6 \times 1 \mathrm{k}$ mesh for geometry optimization and with a $12 \times 12 \times 1 k$ mesh for self-consistent electronic structure calculations for band dispersion. Geometry optimization has been performed for all atoms in the slab, until the remaining force acting on atoms is less than $0.005 \mathrm{Ry} / \AA$. The conjugated-gradient minimization scheme is used for both the electronic structure calculation and for the geometry optimization.

The surfaces are simulated using a repeated-slab model in which $9 \mathrm{Si}$ atomic layers of $\alpha$ quartz, graphene, and a $10 \AA$-vacuum region are included. We chose (0001) 

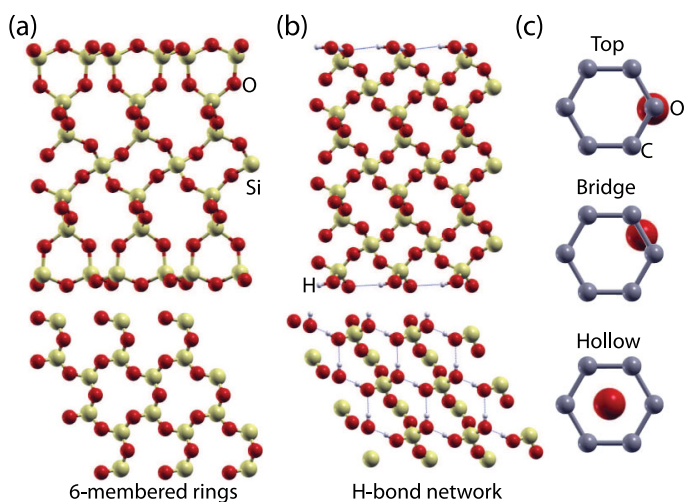

FIG. 1 (color online). Side and top views of reconstructed structures of (a) O-terminated $\mathrm{SiO}_{2}$ (0001) surface, and (b) fully hydroxylated $\mathrm{SiO}_{2}$ (0001) surface. The hydrogen bonds are shown in dashed lines. (c) Three representative arrangements of graphene on $\mathrm{SiO}_{2}$ : top $(T)$ configuration, bridge $(B)$ configuration, and hollow $(H)$ configuration.

surfaces of $\alpha$ quartz as a model surface of $\mathrm{SiO}_{2}$ on which to adsorb the graphene. To avoid periodic image errors arising from the slab model, we adopt the effective-screening medium method $[22,23]$. We do not consider simple cleaved surfaces (as used for previous calculations) [14-16] but fully optimized oxygen-terminated (0001) surfaces to simulate experimentally relevant situations. Previous experimental and theoretical studies [24,25] have shown that the upper or lower two subsurfaces of cleaved $\mathrm{SiO}_{2}$ undergo surface reconstruction at around $300 \mathrm{~K}$ to give O-terminated surfaces with six-membered rings as shown in Fig. 1(a). Besides these O-terminated surfaces, cleaved surfaces will also be rapidly hydroxylated under ambient conditions, yielding silanol groups (Si-OH) [25-27]. Thus, in this Letter, we also consider the fully hydroxylated $\mathrm{SiO}_{2}$ surface possessing a zigzag hydrogen bonded network [Fig. 1(b)]. On these surfaces, we put graphene with $2 \times 2$ lateral periodicity to keep the commensurability condition with the lateral cell of $\mathrm{SiO}_{2}$ (less than $0.08 \%$ lattice mismatch). To investigate how the energetics and electronic structure of graphene depend on the local configuration on $\mathrm{SiO}_{2}$, we considered three representative arrangements of graphene on $\mathrm{SiO}_{2}$ where an oxygen atom (from either the reconstructed O-terminated or hydroxylated surface) is situated directly below either: a $\mathrm{C}$ atom, the center of a $\mathrm{C}-\mathrm{C}$ bond, or the hollow site at the center of the $\mathrm{C}$ hexagonal ring, which are classified as the top $(T)$, the bridge $(B)$, and the hollow $(H)$ configurations, respectively, as shown in Fig. 1(c).

Figures 2(a)-2(c) show the fully optimized geometric structures of graphene adsorbed on O-terminated $\mathrm{SiO}_{2}$ surfaces. In all cases, graphene keeps its plane and hexagonal atomic network, maintaining the spacing of $2.9 \AA$ for $T, B$, and $H$ configurations, when bound to the $\mathrm{SiO}_{2}$ surfaces. Of the three configurations, the hollow one is the most stable. The calculated binding energy per $\mathrm{C}$ atom of

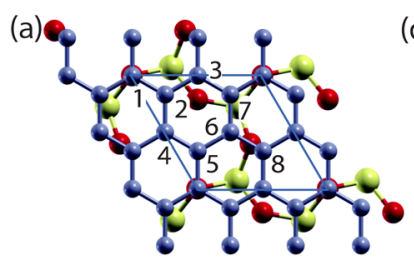

(b)

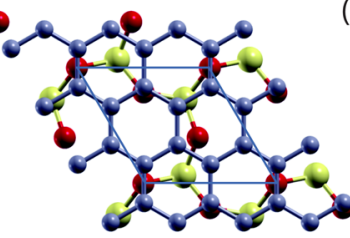

(e)
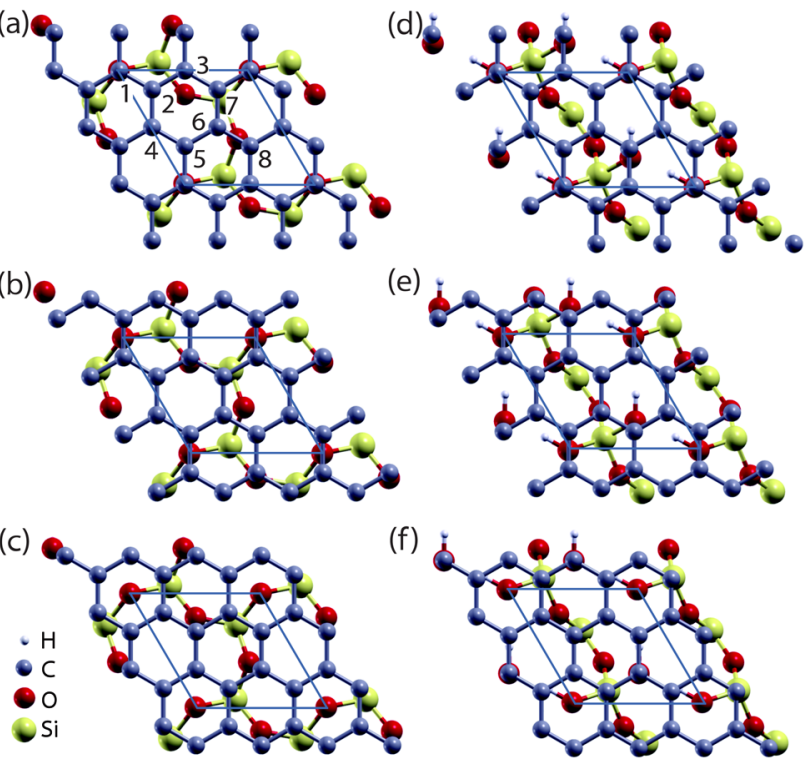

(f)



FIG. 2 (color online). Top views of optimized structures of graphene on (a) top, (b) bridge, (c) hollow sites of O-terminated $\mathrm{SiO}_{2}$ (0001) surface, and on (d) top, (e) bridge, (f) hollow sites of fully hydroxylated $\mathrm{SiO}_{2}$ (0001) surface. The surface unit cell is shown by the solid lines. The indexes of $\mathrm{C}$ atoms in the unit cell are used in the Tables III and IV.

$H$ configuration is $15 \mathrm{meV}$, about $3 \mathrm{meV}$ greater than that of the other two configurations (Table I). These results indicate that the energetics of graphene on O-terminated surfaces are not very sensitive to the adsorption site [28].

We also find similar optimized structures and energetics for graphene on fully hydroxylated $\mathrm{SiO}_{2}$ surface. As shown in Figs. 2(d)-2(f), graphene essentially retains its initial structure with separations of about $2.93 \AA$ for all configurations. The binding energies of graphene on hydroxylated surfaces are 8, 10.7, and $13 \mathrm{meV}$ for $T, B$, and $H$ configurations, respectively. Thus, as for the O-terminated surface, graphene is loosely bound to the surface without any site selectivity.

These energetics of adsorption of graphene on both O-terminated and hydroxylated surfaces correspond well

TABLE I. Equilibrium distance $d_{0}(\AA)$ and binding energy $E_{b}$ (meV/C atom) of graphene on different adsorption sites of O-terminated and hydroxylated $\mathrm{SiO}_{2}$ (0001) surfaces. Equilibrium distance $d_{0}$ is the distance between graphene layer and topmost atom in $\mathrm{SiO}_{2}$ slab. The $E_{b}$ is estimated by the equation $E_{b}=E_{G}+E_{\mathrm{SiO}}-E_{G / \mathrm{SiO}}$, where $E_{G}, E_{\mathrm{SiO}}$, and $E_{G / \mathrm{SiO}}$ are total energies of the isolated graphene, isolated $\mathrm{SiO}_{2}$ slab, and graphene- $\mathrm{SiO}_{2}$ hybrid systems, respectively.

\begin{tabular}{lcccr}
\hline \hline & \multicolumn{2}{c}{ O terminated } & \multicolumn{2}{c}{ Hydroxylated } \\
Adsorption site & $d_{0}$ & $E_{b}$ & $d_{0}$ & $E_{b}$ \\
\hline Top & 2.88 & 11.1 & 2.96 & 8.3 \\
Bridge & 2.90 & 10.9 & 2.93 & 10.7 \\
Hollow & 2.89 & 14.6 & 2.91 & 12.8 \\
\hline \hline
\end{tabular}


(a)

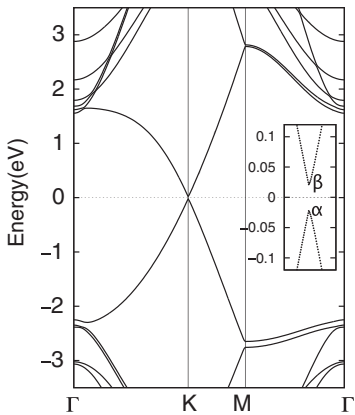

(b)

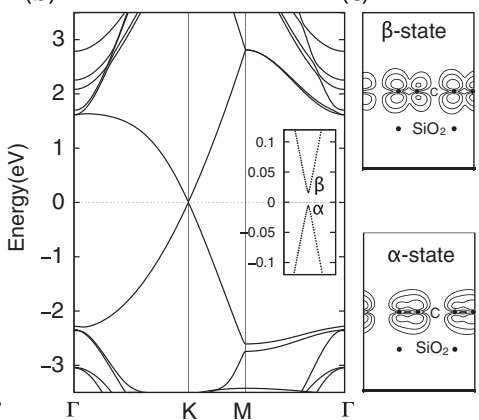

TABLE III. Electrostatic potential of each $\mathrm{C}$ atom of graphene on O-terminated (0001) surface of $\mathrm{SiO}_{2}$. The energy (meV) is measured from the electrostatic potential of $\mathrm{C}$ atom in isolated graphene. Indexes correspond with the $\mathrm{C}$ atomic sites denoted in Fig. 2(a).

\begin{tabular}{lcccccccc}
\hline \hline & $\mathrm{C}_{1}$ & $\mathrm{C}_{2}$ & $\mathrm{C}_{3}$ & $\mathrm{C}_{4}$ & $\mathrm{C}_{5}$ & $\mathrm{C}_{6}$ & $\mathrm{C}_{7}$ & $\mathrm{C}_{8}$ \\
\hline Top & 69 & 67 & 71 & 78 & 69 & 81 & 63 & 58 \\
Bridge & 44 & 74 & 51 & 51 & 77 & 51 & 73 & 73 \\
Hollow & 56 & 61 & 70 & 51 & 74 & 69 & 63 & 63 \\
\hline \hline
\end{tabular}

FIG. 3. Electronic energy band of graphene on (a) O-terminated $\mathrm{SiO}_{2}$ (0001) surface, and on (b) hydroxylated $\mathrm{SiO}_{2}(0001)$ surface. The insets are expansions of the bands around the $K$ point. (c) Contour plots of the square wave function of the states denoted by $\alpha$ and $\beta$ in the insets.

with the previous experimental work [29], in which the binding energy was determined to be $16 \mathrm{meV}$ per $\mathrm{C}$ atom. However, the spacing between the graphene and the surfaces was about $1.3 \AA$ larger than those spacings obtained in our calculations. This structural discrepancy probably arises from the difference between the model $\mathrm{SiO}_{2}$ structure used in the calculation and the amorphous $\mathrm{SiO}_{2}$ used in the experiment. For instance, the nanometer scale structures on $\mathrm{SiO}_{2}$, such as steps and adsorbates of environmental materials, lead the larger spacing between graphene and substrates. It must be pointed out that our results are inconsistent with previous theoretical results, in which a graphene monolayer is found to bound strongly to O-terminated $\mathrm{SiO}_{2}$ surfaces via covalent bonds [14-16]. In the previous calculations, graphene has been adsorbed on cleaved $\mathrm{SiO}_{2}$ surfaces which have unsaturated $\mathrm{O}$ dangling bonds that can easily form a covalent $\mathrm{CO}$ bond, and hence lead to the different energetics and adsorbed structures. It has been experimentally established that graphene is weakly bound to $\mathrm{SiO}_{2}$ substrates via van der Waals and/ or capillary forces [1]. Therefore, our results on energetics and atomic structures agree with the experimental results. From our calculations, we also estimate the energy cost for the corrugation of graphene as a few meV per $\mathrm{C}$ atom. Thus, the interaction between graphene and $\mathrm{SiO}_{2}$ surfaces is sufficient to overcome the energy cost for the corrugation of graphene. In other words, the structure of graphene follows the morphology of the $\mathrm{SiO}_{2}$ substrates.

TABLE II. Band gap (meV) of monolayer graphene and bilayer graphene on different adsorption sites of O-terminated and hydroxylated (0001) surfaces of $\mathrm{SiO}_{2}$.

\begin{tabular}{lcccc}
\hline \hline & \multicolumn{2}{c}{ O terminated } & \multicolumn{2}{c}{ Hydroxylated } \\
Adsorption site & Monolayer & Bilayer & Monolayer & Bilayer \\
\hline Top & 44 & 4 & 11 & 1 \\
Bridge & 29 & 1 & 13 & 0 \\
Hollow & 18 & 6 & 23 & 0 \\
\hline \hline
\end{tabular}

Next, we focus on the electronic structures of graphene on the $\mathrm{SiO}_{2}$ surfaces. Figures 3(a) and 3(b) show the electronic energy band of the graphene adsorbed on the O-terminated and hydroxylated (0001) surfaces of $\mathrm{SiO}_{2}$. On both $\mathrm{SiO}_{2}$ surfaces, the character of the electronic energy band of pristine monolayer graphene seems to be preserved. However, focusing on the Dirac cone at the $K$ point, reveals that the $\pi$ and $\pi^{*}$ bands repulse each other, forming a small energy gap at the $K$ point as shown in the insets of Figs. 3(a) and 3(b). Thus, the graphene adsorbed on the $\mathrm{SiO}_{2}$ surfaces is no longer metallic with massless electrons, but semiconducting with a direct narrow fundamental gap. Table II lists the calculated gaps of graphene with different adsorption sites on O-terminated and hydroxylated $\mathrm{SiO}_{2}$ surfaces. We find that the band gaps of graphene on the O-terminated $\mathrm{SiO}_{2}$ (0001) surface are larger than those on the fully hydroxylated $\mathrm{SiO}_{2}$ surfaces. The band gaps are found to be $11-44 \mathrm{meV}$ depending on the adsorption arrangement of graphene and the surface structures of $\mathrm{SiO}_{2}$, despite the energetics and geometric structure of graphene on $\mathrm{SiO}_{2}$ being largely independent of adsorption arrangement. These gap values are comparable to the band gaps obtained for graphene on hexagonal boron nitride or $\mathrm{Cu}(111)$ surface [30,31]. Furthermore, the values are significantly higher than $k_{B} T$ at room temperature so that the adsorption decreases the transport properties of graphene compared with the free standing graphene.

The semiconducting properties of graphene on $\mathrm{SiO}_{2}$ imply that the on-site energy of $\mathrm{C}$ atoms is modulated by the adsorption and depends on the mutual arrangement relavtive to the $\mathrm{O}$ atoms on the surfaces. To further investigate this, we analyzed the electrostatic potential of $\mathrm{C}$ atoms of graphene on $\mathrm{SiO}_{2}$ surfaces. We found that there

TABLE IV. Electrostatic potential of each $\mathrm{C}$ atom of graphene on hydroxylated (0001) surface of $\mathrm{SiO}_{2}$. The energy $(\mathrm{meV})$ is measured from the electrostatic potential of $\mathrm{C}$ atom in isolated graphene. Indexes correspond with the $\mathrm{C}$ atomic sites denoted in Fig. 2(a).

\begin{tabular}{lcccrcrcc}
\hline \hline & $\mathrm{C}_{1}$ & $\mathrm{C}_{2}$ & $\mathrm{C}_{3}$ & $\mathrm{C}_{4}$ & $\mathrm{C}_{5}$ & $\mathrm{C}_{6}$ & $\mathrm{C}_{7}$ & $\mathrm{C}_{8}$ \\
\hline Top & 120 & 126 & 119 & 142 & 134 & 144 & 127 & 117 \\
Bridge & 103 & 124 & 108 & 96 & 110 & 95 & 128 & 109 \\
Hollow & 104 & 137 & 104 & 126 & 163 & 142 & 136 & 161 \\
\hline \hline
\end{tabular}





FIG. 4. Electronic energy band of bilayer graphene on (a) O-terminated $\mathrm{SiO}_{2}$ (0001) surface, and on (b) hydroxylated $\mathrm{SiO}_{2}(0001)$ surface. The insets are expansions of bands around the $K$ point.

is spatial inhomogeneity in the electrostatic potential of $\mathrm{C}$ atoms of graphene. Indeed, the electrostatic potential on graphene adsorbed on $\mathrm{SiO}_{2}$ strongly depends on the $\mathrm{C}$ sites and is shallower by $59-100 \mathrm{meV}$ than that of $\mathrm{C}$ atoms belonging in isolated graphene [Tables III and IV]. The variation of the electrostatic potential is 23 and $45 \mathrm{meV}$ for graphene on O-terminated and hydroxylated $\mathrm{SiO}_{2}(0001)$ surfaces with on-top arrangements, respectively. These values are the same order as the gap energy of graphene on $\mathrm{SiO}_{2}$. Indeed, single orbital tight-binding calculations for isolated graphene with on-site energy variation result in the energy gap of $10 \mathrm{meV}$ at $K$ point. Thus, we conclude that the variation in electrostatic potential or on-site energy of $\mathrm{C}$ atoms disrupts the degeneracy of the $\pi$ and $\pi^{*}$ bands at the $K$ point resulting in their semiconducting character. The on-site energy difference can be observed in the wave functions from the top of valence band [ $\alpha$ state in Fig. 3(c)] and the bottom of the conduction band [ $\beta$ state in Fig. 3(c)] at the $K$ point. The distribution of the wave function of the $\pi$ state in graphene is not equivalent between adjacent $\mathrm{C}$ atoms as in the case of hexagonal boron nitride which exhibit $\pi$ bonding character. The inhomogeneous on-site energy of the $\mathrm{C}$ atoms is induced not by the charge transfer between the graphene and the surfaces but by the inhomogeneous charge distribution on the $\mathrm{SiO}_{2}$ surfaces. Indeed, our analyses of charge distribution show that there is no charge transfer to or from graphene. The charge redistribution occurs only at the interfacial region, and induces very small dipole moments.

Next, we investigate the electronic properties of bilayer graphene on $\mathrm{SiO}_{2}$ surfaces. We find that the equilibrium interlayer distance with $A B$ stacking arrangement is $3.35 \AA$ A. Figures 4(a) and 4(b) show the electronic energy band of bilayer graphene adsorbed on O-terminated and hydroxylated $\mathrm{SiO}_{2}$ surfaces. These systems exhibit the parabolic bands around the Fermi level which resemble those of pristine bilayer graphene. Detailed analyses of the electronic energy bands around the Fermi level revealed that a small band gap of less than $6 \mathrm{meV}$ is still opened [see the inset of Fig. 4(a)]. The band gap values of bilayer graphene with different configurations on both O-terminated and hydroxylated $\mathrm{SiO}_{2}$ surfaces are listed in Table II. However, these band gaps are within the accuracy limitation of the LDA, and therefore we can assume that the band gap value is zero. Thus, the zero gap of the graphene layer is recovered when a second graphene layer is placed.

This work was partly supported by CREST, Japan Science and Technology Agency, and a Grant-in-Aid for scientific research from the Ministry of Education, Culture, Sports, Science and Technology of Japan.

[1] K. S. Novoselov et al., Science 306, 666 (2004).

[2] A. K. Geim and K.S. Novoselov, Nature Mater. 6, 183 (2007).

[3] I. Forbeaux, J.-M. Themlin, and J.-M. Debever, Phys. Rev. B 58, 16396 (1998).

[4] C. Berger et al., Science 312, 1191 (2006).

[5] K. S. Novoselov et al., Nature (London) 438, 197 (2005).

[6] Y. Zhang et al., Nature (London) 438, 201 (2005).

[7] M. Otani et al., Phys. Rev. B 81, 161403(R) (2010).

[8] J. B. Oostinga et al., Nature Mater. 7, 151 (2007).

[9] Y. Zhang et al., Nature (London) 459, 820 (2009).

[10] M. F. Craciun et al., Nature Nanotech. 4, 383 (2009).

[11] T. Ohta et al., Science 313, 951 (2006).

[12] S. Y. Zhou et al., Nature Mater. 6, 770 (2007).

[13] A. Mattausch and O. Pankratov, Phys. Rev. Lett. 99, 076802 (2007).

[14] Y.-J. Kang, J. Kang, and K. J. Chang, Phys. Rev. B 78, 115404 (2008).

[15] M.Z. Hossain, Appl. Phys. Lett. 95, 143125 (2009).

[16] P. Shemella and S. K. Nayak, Appl. Phys. Lett. 94, 032101 (2009).

[17] P. Hohenberg and W. Kohn, Phys. Rev. 136, B864 (1964).

[18] W. Kohn and L. J. Sham, Phys. Rev. 140, A1133 (1965).

[19] D. M. Ceperley and B. J. Alder, Phys. Rev. Lett. 45, 566 (1980).

[20] J. P. Perdew and A. Zunger, Phys. Rev. B 23, 5048 (1981).

[21] D. Vanderbilt, Phys. Rev. B 41, 7892 (1990).

[22] M. Otani and O. Sugino, Phys. Rev. B 73, 115407 (2006).

[23] I. Hamada et al., Phys. Rev. B 80, 165411 (2009).

[24] G.-M. Rignanese et al., Phys. Rev. B 61, 13250 (2000).

[25] T. P. M. Goumans et al., Phys. Chem. Chem. Phys. 9, 2146 (2007).

[26] G.-M. Rignanese et al., Phys. Chem. Chem. Phys. 6, 1920 (2004).

[27] J. Yang and E. G. Wang, Phys. Rev. B 73, 035406 (2006).

[28] It should be noted that LDA well reproduces the interunit spacing of van der Waals materials.

[29] M. Ishigami et al., Nano Lett. 7, 1643 (2007).

[30] G. Giovannetti et al., Phys. Rev. B 76, 073103 (2007).

[31] S. Okada, Jpn. J. Appl. Phys. 49, 020204 (2010). 